\title{
Using Stroke-Number-Characteristics for Improving Efficiency of Combined Online and Offline Japanese Character Classifiers
}

\author{
Ondrej Velek and Masaki Nakagawa \\ Tokyo University of Agr. \& Tech. 2-24-16 Naka-cho, Koganei-shi, Tokyo 184-8588, Japan \\ velekehands.ei.tuat.ac.jp
}

\begin{abstract}
We propose a new technique for normalizing likelihood of multiple classifiers prior to their combination. During a combination process we utilize the information about their efficiency correctly recognize a character with a given stroke number. In the beginning, we show that this recognizer's efficiency based on a stroke number is different for a common on-line and offline recognizer. Later, we demonstrate on elementary combination rules, such as sum-rule and max-rule that using this information increases a recognition rate.
\end{abstract}

\section{Introduction}

Combining different classifiers for the same classification problems has become very popular during the last years [1]. The bigger difference among classifiers promises better improvement of a recognition rate, which can be achieved by their combination. To take an advantage of the difference between on-line and off-line classifiers seems be a good idea: on-line recognition can use valuable information of writing order and has no problem with segmentation of overlapped strokes, complementary to it, off-line recognition guarantees robustness against stroke order and stroke number variations, which is usually the main disadvantage of on-line systems.

However, combination of two independent off/on-line classifiers brings the problem, how to decide which result of which classifier is correct. Our approach is based on well-known fact that a specialization is good way to achieve improvement. Let's suppose that an off-line recognizer is better for recognizing some characters than an on-line recognizer. We try to find an identifiable characteristic of characters, which can divide unknown input character patterns to several groups. We will investigate which recognizer is more efficient for correct classifying to each of these classes and in a combination scheme we will utilize this additive information. In this paper, we investigate a characteristic of on-line and off-line Japanese character classifiers in dependency on number of strokes.

\section{Characteristic of Classifiers According to Stroke Number}

Number of stroke is the basic feature of each Chinese or Japanese character. The right stroke number can be found in a dictionary and varied from 1 to about 30 . However, for fluently and quickly written characters, number of strokes is often lower, because 
some strokes are connected. In some cases number of strokes can be higher than should be. An interesting characteristic of a variation in stroke numbers is in [2].

Let's see a recognition rate according to a stroke number for one on-line and two off-line recognizers Graph 1. Even from a brief view, we see the big difference between on-line and off-line classifiers. Both off-line recognizers are weaker in recognizing characters written by a low number of strokes. The recognition rate simultaneously grows with increasing complexity of patterns. It's in accordance to well-known fact that for off-line classifier it's more difficult to recognize simple characters like kana than difficult kanji. On the contrary, on-line recognizer is hiefficient for patterns written by one stroke. We see that although an average rate of the on-line recognizer is about $5 \%$ worst than that of the off-line recognizer, for characters written by one, two and three strokes a recognition rate is better, or at least similar. In the rest of this paper we will try to utilize this feature in a combination scheme.

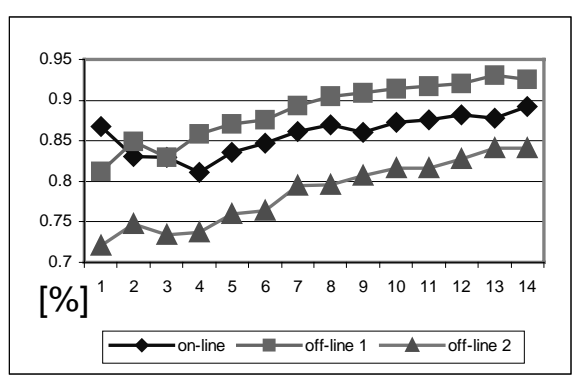

Graph 1. Recognition rate according to a stroke number for three recognizers.

\section{Combining Classifiers}

A typical output of each recognizer is several candidates and for each candidate also his likelihood, which can merely be approximations of the correct aposteriori probabilities. In [3] we have introduced a new warping technique for normalizing likelihood of multiple classifiers, so that likelihood can be easily compared. Our technique takes classifier-specific likelihood characteristics into account and maps them to a common, ideal characteristic allowing fair combination. For each classifier we create an accumulated characteristic function, which express an a-posteriori probability $\mathrm{P}\left(\left\langle 0, a_{\mathrm{i}}\right\rangle\right)$ that for each interval $\left\langle 0, a_{\mathrm{i}}\right\rangle, a_{\mathrm{i}}$ is an output value of a recognizer, an input pattern was recognized correctly. An accumulated recognition rate is a continuous, monotone growing function over a classifier's output.

We normalize the output of each single recognizer so that the accumulated probability function $\mathrm{P}\left(<0, a_{\mathrm{i}}>\right)$ becomes a linear function proportional to the classifier output. Accordingly, we normalize each classifier output by adding an adjustment: $a_{i}^{\prime}=a_{i}+\operatorname{charf}\left(a_{i}\right)$ with

$$
\operatorname{charf}\left(a_{i}\right)=a_{\max } * R_{i}-a_{i}=a_{\max } * \frac{\sum_{k=0}^{i} n_{\text {correct }}(k)}{N}-a_{i}
$$

where $a_{\max }$ is the maximum possible output of a classifier ( $a_{\max }=1,000$ in our experiments), $\mathrm{N}$ is the number of overall patterns, and $\mathrm{R}_{\mathrm{i}}$ stands for the partially accumulated recognition rate. We call this classifier-specific adjustment the characteristic function $\left[\right.$ charf $_{i}$ ] of a classifier. In our previous work, we calculated one characteristic function for each classifier. Now we will create not one, but many different characteristic functions, one for characters written by the same number of strokes. In this paper we have 14 functions, where the last one is for patterns written 
by fourteen or more strokes. In Graph 2 we see characteristic function [charf $]$ for our on-line recognizer and in Graph 3 for an off-line recognizer.

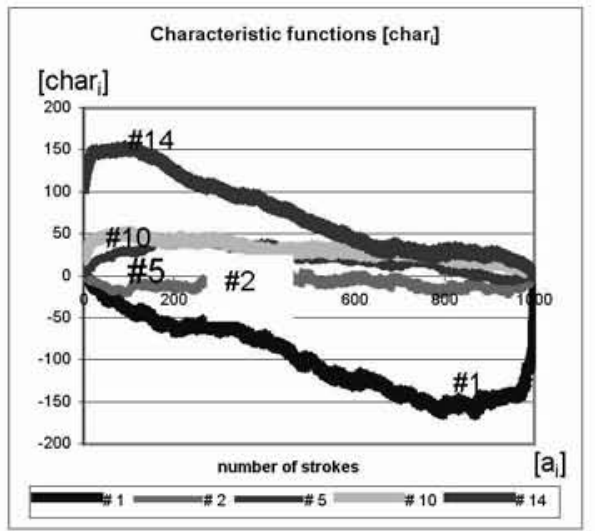

Graph 2. Characteristic function for online recognizer

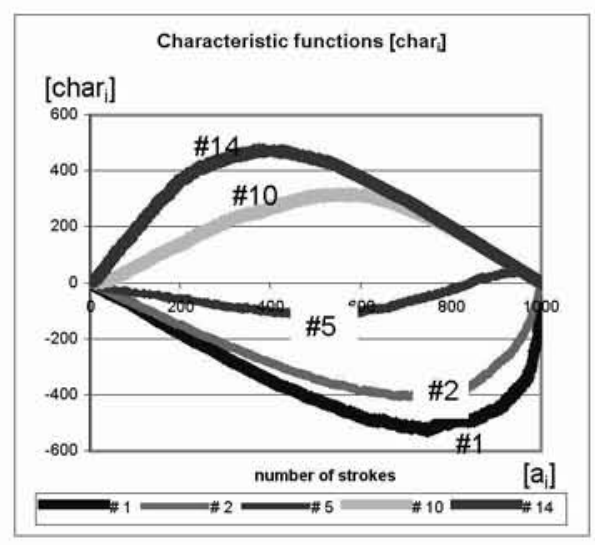

Graph 3. Characteristic function for offline_1 recognizer.

\section{Classifiers and Database Used in Experiments}

Off-line_1 recognizer is based on MQDF, while off-line_2 recognizer on LVQ. More information about these two off-line recognizers and also about our On-line recognizer is written in our previous paper [3].

We use NTT-AT database, which contains data written by elderly people with an average age of 70.5 years; with the oldest writer being 86 years old. The patterns contained in NTT-AT are casually written, very often with an untypical stroke order. We have chosen this database because it is difficult to recognize for many recognizers. We also consider it a very good test bed for underpinning our conjecture saying that combining on-line classifiers with stroke-order independent off-line classifiers leads to better overall recognition rates. From this database we generate a dual off-line version using our method for generating realistic Kanji character images from on-line patterns [4]. This method combines on-line patters with a calligraphic stroke shape library, which contains genuine off-line patterns written with different writing tools. Since the artificially generated off-line images are combinations of actual on-line and off-line patterns they look very natural and realistic. Thanks to this duality, an on-line database can be used by off-line as well as an on-line classifier.

\section{Experimental Results}

We used two different combination strategies for combining our on-line and off-line recognizer: max-rule and sum-rule. The max-rule takes the class with the maximum output value among each classifier, while the sum-rule sums up the output for each 
class and selects the one with the highest sum. These rules are motivated by a paper by Kittler et al. [5], which gives a theoretical explanation for the frequently observed superiority of the sum-rule. We have added also AND and OR rules, which means that pattern was recognized by both recognizers, or respectively at least by one. These rules cannot be used in real application, but they show us the worst and the best possible combination strategy. The first row of Table 1 shows combination without normalization of recognizer's likelihood. The second row shows result after applying a common characteristic function [3], and in the last row there are results after employing 14 characteristics functions, one for each group of characters with the same stroke number.

Table 1. Combination of one on-line and one off-line recognizer

\begin{tabular}{|l|r|r|r|r|}
\hline & \multicolumn{1}{|c|}{ AND } & \multicolumn{1}{c|}{ OR } & Maximum & \multicolumn{1}{c|}{ Sum } \\
\hline without normalization & 79.03 & 95.71 & 92.04 & 92.08 \\
\hline normalization 1 characteristic & 79.03 & 95.71 & 92.11 & 93.40 \\
\hline normalization 14 characteristics & 79.03 & 95.71 & 92.19 & 93.68 \\
\hline
\end{tabular}

\section{Discussion}

We have demonstrated a new method for combining different classifiers with a various range of output likelihood. Unlike our previous method [3], where only one characteristic is for a recognizer, in our new approach we study recognizer's characteristic in more details and we make 14 different characteristic functions in dependency on number of strokes. By applying this, we have got further improvement of recognition rate. Advantage of this method is that the realization doesn't consume additive time during the recognition process and only minimal additive space for saving more characteristic functions. Making characteristic function is done only once, after training recognizer. From [3] we know, that our normalization method is more beneficial, if more classifiers are combined, so next time we want to employ more than two classifiers. We are also experimenting with another characteristics, not only these based on different number of strokes. If we find another characteristic, for which the difference between on-line and off-line classifier is bigger, we can more utilize advantage of each single classifier.

\section{References}

[1] H. Tanaka, K. Nakajima, K. Ishigaki, K. Akiyama, M. Nakagawa, Hybrid Pen-Input Character Recognition System Based on Integration of On-Line - Off-Line Recognition, $5^{\text {th }}$ ICDAR (1999) 209-212

[2] K. Matsumoto, T. Fukushima, M. Nakagawa, Collection and analysis of on-line handwritten Japanese character patterns, Proc. 6th ICDAR, Seattle, 2001, pp.496-500.

[3] O. Velek, S. Jaeger, M. Nakagawa, A warping technique for normalizing likelihood of multiple classi-fiers and its effectiveness in combined on-line/off-line Jap. char.recognition, accepted for IWFHR 2002

[4] O. Velek, Ch. Liu, M. Nakagawa, Generating Realistic Kanji Character Images from Online Patterns, Proc. 6th ICDAR (2001) 556-560

[5] J.Kittler, M.Hatef, R.Duin, J.Matas, On Combining Classifiers, IEEE PAMI 20(3) (1998) 222-239 